\title{
Capacitação para o exercício da preceptoria pelo enfermeiro na Residência Multiprofissional em Saúde
}

\author{
Training for the exercise of preceptorship by nurses in the Multiprofessional Residency in \\ Health
}

\author{
Formación para el ejercicio de la preceptoría por enfermeros en la Residencia \\ Multiprofesional en Salud
}

Verônica Caé da Silva ${ }^{1,2 *}$, Ligia de Oliveira Viana ${ }^{3}$, Alexandra Schmitt Rasche ${ }^{3}$, Pacita Geovana Gama de Sousa Aperibense ${ }^{3}$, Audrei Castro Telles ${ }^{2,4}$, Daniela de Oliveira Matias ${ }^{5,6}$.

\begin{abstract}
RESUMO
Objetivo: Descrever a capacitação do enfermeiro para o desenvolvimento da preceptoria na Residência Multiprofissional em Saúde (RMS). Métodos: Recorte da tese de doutorado "Os saberes que emergem da prática social do enfermeiro-preceptor na residência multiprofissional em saúde", de 2017. Estudo de caso único, de uma instituição de saúde do Rio de Janeiro (RJ). Foram entrevistados 46 participantes de 2 programas de RMS, analisados e categorizados os resultados em unidades com análise temática. Resultados: A capacitação para atuar na RMS passa por vivências peculiares, de acordo com o cenário, tempo de formação profissional, o interesse, o envolvimento, o incentivo, o tempo na RMS, as questões que experimentou com os residentes e demais protagonistas e a instrumentalização para esta prática. Os depoentes apontaram como essencial um profissional atualizado, que tenha apropriação de um conteúdo mínimo de saberes do campo da educação para além dos atributos pessoais, como o gostar de ensinar e dos profissionais, ser um excelente enfermeiro assistencial. Tal aprendizado pode ocorrer por meio de cursos de capacitação para preceptoria. Conclusão: $O$ enfermeiro-preceptor além de ter experiência na área, precisa de capacitação permanente para desenvolver as ações junto aos residentes no cenário vivo dos serviços de saúde.
\end{abstract}

Palavras-chave: Enfermagem, Ensino, Preceptoria, Internato não médico.

\begin{abstract}
Objective: To describe the training of nurses for the development of preceptorship in the Multiprofessional Residency in Health (RMS). Methods: Clipping of the doctoral thesis "The knowledge that emerges from the social practice of the nurse-preceptor in the multiprofessional residency in health", from 2017. Single case study, from a health institution in Rio de Janeiro (RJ). 46 participants of 2 RMS programs were interviewed, the results were analyzed and categorized in units with thematic analysis. Results: The training to work in the RMS goes through peculiar experiences, according to the scenario, professional training time, interest, involvement, encouragement, time in the RMS, the issues that it experienced with residents and other protagonists and the instrumentalization for this practice. The deponents pointed out as essential an updated professional, who has a minimum content of knowledge in the field of education in addition to personal attributes, such as enjoying teaching and the professionals, being an excellent care nurse. Such learning can occur through training courses for preceptorship. Conclusion: The nurse-preceptor, in addition to being have experience in the area, needs permanent training to develop actions with residents in the living scenario of health services.
\end{abstract}

Keywords: Nursing, Teaching, Preceptorship, Internship nonmedical.

\footnotetext{
${ }^{1}$ Hospital Federal de Bonsucesso, Rio de Janeiro - RJ.

2 Prefeitura Municipal de Duque de Caxias, Duque de Caxias - RJ. *E-mail: vcae@hotmail.com

3 Universidade Federal do Rio de Janeiro (UFRJ), Rio de Janeiro - RJ.

4 Instituto Nacional do Câncer, Rio de Janeiro - RJ.

${ }^{5}$ Hospital Federal Cardoso Fontes, Rio de Janeiro - RJ.

${ }^{6}$ Hospital Naval Marcílio Dias, Rio de Janeiro - RJ.
} 


\section{RESUMEN}

Objetivo: Describir la formación de enfermeros para el desarrollo de la preceptoría en la Residencia Multiprofesional en Salud (RMS). Métodos: Recorte de la tesis doctoral "El conocimiento que surge de la práctica social del enfermero-preceptor en la residencia multiprofesional en salud", de 2017. Estudio de caso único, de una institución de salud en Rio de Janeiro (RJ). Se entrevistaron 46 participantes de 2 programas de RMS, los resultados se analizaron y categorizaron en unidades con análisis temático. Resultados: La formación para trabajar en la RMS pasa por experiencias peculiares, según el escenario, tiempo de formación profesional, interés, implicación, estímulo, tiempo en la RMS, las problemáticas que vivió con los residentes y otros protagonistas y la instrumentalización para ello práctica. Los declarantes señalaron como imprescindible un profesional actualizado, que tenga un contenido mínimo de conocimientos en el campo de la educación además de atributos personales, como disfrutar de la docencia y de los profesionales, ser un excelente enfermero de cuidados. Este aprendizaje puede ocurrir a través de cursos de formación para la preceptoría. Conclusión: La enfermera-preceptora, además de tener experiencia en el área, necesita una formación permanente para desarrollar acciones con los residentes en el escenario de vida de los servicios de salud.

Palabras clave: Enfermería, Enseñanza, Preceptoria, Internado no médico.

\section{INTRODUÇÃO}

A Residência Multiprofissional em Saúde (RMS) é conceituada como um modo de formação que congrega pessoas de diferentes categorias e áreas profissionais como: Biologia, Biomedicina, Nutrição, Enfermagem, Serviço Social, Odontologia, Psicologia, Farmácia, Educação Física, Medicina Veterinária, Fisioterapia, Fonoaudiologia e Terapia Ocupacional (BRASIL, 1998). Trata-se de uma modalidade de experiência prática remunerada em serviço e está voltada, em sua maioria, aos recém-graduados.

Em termos conceituais, a residência é uma forma de treinamento em serviço, que baseia-se na aprendizagem pela ação prática nos cenários de saúde nacionais. As atividades dos residentes são realizadas com cunho intensivo para a aquisição de competências e habilidades técnicas- assistenciais, gerenciais, educacionais e de relacionamento com os usuários, familiares e os próprios profissionais do serviço, em determinada área específica de conhecimento (SILVA VC, 2017).

A atuação dos graduados como residentes pode se firmar em termos legais nos moldes de treinamento profissional in loco ou estar atrelada a um Curso de Pós-graduação Lato Sensu, tendo o estudante que assumir o compromisso de frequentar disciplinas teóricas, prestar assistência à saúde aos indivíduos num cenário de cuidado, participar de encontros com mestres e ou doutores para orientação e apresentar um trabalho de conclusão ao final. O cumprimento de todas as etapas levará a certificação na área prática vivenciada, conforme legislação vigente de cada Conselho Federal Profissional, durante a duração mínima de dois anos previstos de atividade (BRASIL, 2012).

Assim, acompanhando os movimentos sócio-político-econômicos de reestruturação da saúde pública e as diretrizes educacionais no país do Conselho Nacional de Educação (CNE), com destaque para a formação de novos profissionais para o Sistema Único de Saúde (SUS), em 2005, foi promulgada a Lei oㅜ 11.129, que criou as RMS (BRASIL, 2005). Sua criação buscava atender às questões específicas e necessidades de formação e educação para e pelo trabalho.

Quanto à preceptoria esta pode ser compreendida como a atuação do profissional de nível superior de saúde nas seguintes etapas: planejamento, construção do Projeto Pedagógico (PP), organização das atividades, supervisão direta e avaliação do desempenho contínuo dos estudantes de graduação e pósgraduação, durante a vivência de práticas de cuidado em saúde voltadas à população (SILVA VC, 2017).

Para acompanhar os residentes em suas atividades, o preceptor deve ser um profissional com vínculo empregatício na instituição e neste sentido, espera-se que seja um profissional habilitado, especialista na área de concentração na qual o residente vivenciará as ações de saúde, ou com tempo de serviço que configure e solidifique a posição de experiente (SILVA VC, et al., 2014; SOUZA SV e FERREIRA BJ, 2019). 
O preceptor deve ter o domínio dos instrumentos teóricos e práticos de sua profissão, pois entende-se ser uma influência positiva, modelo de profissional que colabora, com a prática profissional, no processo educativo e na inserção de novos agentes para o mundo do trabalho (FRANCO TB e MERHY EE, 2012).

Ao assumirem a responsabilidade pela preceptoria, os enfermeiros podem, portanto, vivenciar um processo de transformação pessoal e do seu entorno, no desenvolvimento de um trabalho educativo junto aos residentes. Para tal, é importante que tenham competências e habilidades pedagógicas que facilitem a mediação entre a teoria e a prática na formação dos futuros profissionais especialistas (RIBEIRO PKC, et al.,2020).

O presente artigo aborda as questões que permeiam a atuação do enfermeiro-preceptor em dois programas de RMS - Saúde da Mulher (SM) e Saúde da Família e Comunidade (SFC), buscando responder as questões colocadas, tendo como objetivo: descrever a capacitação do enfermeiro para o desenvolvimento da preceptoria na Residência Multiprofissional em Saúde.

\section{MÉTODOS}

Recorte da tese de doutorado intitulada "Os saberes que emergem da prática social do enfermeiropreceptor na residência multiprofissional em saúde", defendida em 2017 e trata-se de uma pesquisa com abordagem qualitativa, estudo de caso do tipo único integrado, cuja Unidade do Caso foi uma instituição de saúde de uma Universidade Pública, situada no estado do Rio de Janeiro, que possui credenciamento junto aos Ministérios da Saúde e Educação para o desenvolvimento de dois Programas de RMS, que estão recebendo alunos residentes desde 2010 (SILVA VC, 2017).

Os participantes da pesquisa foram quarenta e seis profissionais (chamados também de atores sociais) que compõe os Programas de RMS: treze preceptores, sendo onze enfermeiros - identificados pelas letras "EP", seis tutores - identificados pela letra "T"; quatro coordenadores - identificados pela letra "C"; dois residentes do segundo ano e vinte e um do primeiro ano todos identificados pela letra "R". Após as letras de identificação, registramos o número designativo na ordem em que ocorreram os depoimentos, e, no caso dos residentes, a categoria profissional.

A coleta de dados ocorreu por meio de entrevista e observação, além da análise da documentação dos Programas da RMS. O projeto foi aprovado pelo Comitê de Ética e Pesquisa da Escola de Enfermagem Anna Nery (EEAN), sob o número de Parecer: 776.075 e de CAAE: 34835714.0.0000.5238, em respeito à Resolução no466/2012 do Conselho Nacional de Saúde (BRASIL, 2012).

A análise foi desenvolvida nas seguintes etapas: leitura compreensiva do conjunto do material; exploração e síntese interpretativa (MINAYO MSC, 2007). O referencial utilizado foi a Pedagogia Histórico-Crítica Social dos Conteúdos (SAVIANI D, 2005). Os resultados foram categorizados em duas unidades de análise temática: a percepção do enfermeiro-preceptor sobre sua capacitação para o desenvolvimento das atividades de preceptoria na RMS e a percepção dos demais atores sociais da RMS sobre a capacitação do enfermeiro para o desempenho de suas funções na preceptoria.

\section{RESULTADOS E DISCUSSÃO}

A capacitação do enfermeiro-preceptor para atuar na RMS passa por vivências muito peculiares, dadas a partir do cenário de trabalho, tempo de formação profissional, interesse, envolvimento e incentivo interno e externo para desenvolver a atividade de preceptoria e o tempo de atuação na RMS, as questões que experimentou com os residentes e demais protagonistas dos programas e a instrumentalização para exercício desta prática. Desta forma, a análise dos resultados na unidade caso aqui descrita apontaram:

\section{A percepção do enfermeiro-preceptor sobre sua capacitação para o desenvolvimento das atividades de preceptoria na RMS}

Os enfermeiros-preceptores assumem que precisam de atualização, pois, a correria cotidiana no trabalho dificulta o estudo que se dá de modo mais organizado, programado, sistematizado. Afirmam que a relação com os estudantes permite a aquisição de novos conhecimentos e também é um estímulo para buscar aperfeiçoamento. 
"Querendo ou não o pessoal da universidade está mais atualizado do que quem está na ponta. Porque quem está na ponta está ali naquele dia a dia, não tem muito tempo de chegar em casa e ficar pesquisando o que está rolando de atualização [...]. No máximo, quando chega em casa é para dar uma estudada em algum caso que surgiu durante o dia [...], dar uma atualizada, uma relembrada na matéria ou caso. Enfim, mas, não dá para pesquisar artigos que estão sendo publicados hoje em dia, o que está sendo falado no meio da saúde pública, não sei. Durante a residência eu estava bem mais atualizado do que eu estou agora" (EP4).

"Acho que essa integração com o aluno, com residente, com acadêmico é muito boa para nós, porque quando terminamos a faculdade, ficamos tão imersos nesta rotina de trabalhar, de apagar incêndio, que acabamos nos afastando um pouco disso tudo, por mais que corramos atrás de estudar. Então eles trazem muita coisa para nós. [...] é um jeito de nós estarmos atualizando e buscando também correr atrás. [...] eu acho ótimo" (EP7).

A revisão de matérias e conteúdos que o EP4 relata, para entendimento amplo do atendimento dado a um usuário e continuidade do cuidado não deve ser desconsiderada, pois, agrega conhecimento por meio de instrumentos teóricos, que emergiram após a problematização, identificação de questões que necessitavam de resolução (SAVIANI D, 2005).

Além disso, para manutenção das atividades de instrução e investigação, a integração docenteassistencial deve ser estimulada e propiciada pela universidade e instituições cedentes da RMS, amparando o educador que precisa ser educado (MELLO AL, et al., 2018).

A implementação de um curso de formação para os enfermeiros-preceptores, seja como novidade ou continuidade para os que exercem esta função nas Clínicas da Família e na Instituição de Saúde, atenderia à necessidade evidenciada na análise, destes atores sociais tão atuantes no ensino dos residentes multiprofissionais. Tal fato se constata também pela expressa vontade em se aperfeiçoar:

"Eu estou muito desejosa de fazer o curso de preceptoria, eu acho importantíssimo você fazer o curso de preceptoria, já procurei [...]eu pretendo fazer, eu adoro lidar com estudante" (EP8).

"Existe um curso de preceptoria que hoje é muito difícil de você conseguir fazer, eu já tentei aqui, passei e não tive liberação do serviço para fazer e eu queria muito fazer, um curso [...] eu acho que seria essencial. Acho que o preceptor hoje, ele meio que assume a tarefa e não está tão qualificado para fazer o quanto ele deveria estar. Acho que falta um pouco de base da parte educacional, acho que falta um pouco de base de metodologia [...]" (EP6).

Os enfermeiros-preceptores demonstram que querem aprender, uns até já conhecem os locais que oferecem formação, mas, precisam de liberação para tal sem qualquer prejuízo de ordem financeira e ou do vínculo empregatício, pois, já não são remunerados para a atividade preceptoria e arcar com custos para aprimorar esta ação.

No depoimento a seguir aponta-se que ocorriam reuniões, muito mais frequentes do que atualmente, com a coordenação e equipe responsável pela RMS do programa de SM, onde eram orientados sobre como recepcionar aos residentes e que esse era um modo de serem capacitados para a preceptoria.

"Nós participávamos sempre de reuniões, até mais do que agora. [...] Nessas reuniões [...] nós já éramos orientados da abordagem, de como receber os residentes" (EP1).

A experiência de acompanhar e supervisionar estudantes em nível de graduação e pós-graduação já existia e era exitosa na instituição, em especial na área de atenção à saúde da mulher, mas, o trabalho com os residentes em equipe multiprofissional foi uma novidade difícil de ser vivenciada no início. 
"No início eu achei que estava crua, porque nós nunca tínhamos feito isso, uma multidisciplinar. Já havia feito sim, a residência, mas era só para enfermeiros [...], residência e especialização, que na época era na ginecologia. Mas, que tinha serviço social, psicologia e enfermeiro, nunca tinha estado aqui antes. [...] Entre trancos e barrancos nós fomos tentando aprender todos juntos, a equipe, os residentes e a própria coordenação. Nós vamos tentando aprender e aprendemos cada dia mais [...] Eu acredito que melhorou muito de 2010 para cá" (EP2).

A enfermeira-preceptora relata que permanece aprendendo junto aos demais protagonistas da RMS, permitindo aproximar-se de pessoas em processo de construção da identidade profissional, da linguagem, dos instrumentos e elementos próprios da educação ( $\mathrm{PP}$, folha de frequência, documentos de avaliação, entre outros), acumulando uma importante bagagem em sua história, a ser utilizada na prática com os estudantes multiprofissionais. No entanto, a diferença está em assumir a responsabilidade do ensino de especializados de diversas categorias e a premissa de sentir-se instrumentalizado, desta forma reconhecer o que é próprio dentro de seu espaço de atuação e o que pode e deve ser discutido com os outros, sem extrapolar o limite do exercício profissional (SAVIANI D, 2010).

O trabalho em equipe, tanto dos membros que compõe a RMS quanto dos profissionais do setor onde ocorre a prática clínica, para atender as demandas dos estudantes somada a humildade ao assumir que não são os detentores de todo o conhecimento e estão construindo conhecimento em grupo, foram destacados pelos entrevistados.

"Às vezes eles trazem dúvidas que nós realmente não sabemos tirar. Nós não nos preocupamos em saber tudo, até porque essa área nossa sempre está surgindo coisa nova, sempre está atualizando. Então, como temos o hábito de trabalhar de uma forma como equipe, nós buscamos um ao outro e conseguimos tentar dar conta" (EP3).

Como seres humanos, estamos em progressivo crescimento e aprendizagem, longe de sermos determinados, acabados, completos, assumirmos uma postura de superioridade ou estarmos numa posição de verticalidade na relação com o outro, que é sujeito social ativo na produção da própria existência. Como o homem não é um ser isolado no mundo é importante que se valorize a sua construção histórica, social e coletiva, em especial na esfera educativa (SAVIANI D, 2014).

Alguns enfermeiros indicaram que a prática e o tempo de serviço em dada área qualificam para o desempenho da função de preceptoria na RMS. Cabe reforçar, inclusive, que uma das exigências para ser preceptor é ter experiência clínica na área de formação na qual o residente está se especializando.

"Eu pessoalmente gosto mais da parte prática, do dia a dia. De dizer para eles como é que se faz, poder dar um pouco da minha experiência e aprender" (EP3).

"Eu tenho uma prática, o que me dá a oportunidade de dizer que o que eu tenho eu acho importante repassar. E eu me atrevo a dizer, e vejo hoje o estudante muito carente de pessoas com essa bagagem de assistência" (EP8).

O desejo de aproximação ao aluno, a realização e o prazer no desempenho de suas atividades profissionais, bem como ter conhecimento específico na sua área de atuação são essenciais. $O$ enfermeiropreceptor reconhece que tem a instrumentalização prática, do saber resultante das vivências, que Saviani $D$ (2010) localiza como a forma "sofia" (sabedoria fundada numa longa experiência da vida) de produção do conhecimento. Cabe analisar se também se faz presente a forma "episteme" (a ciência, isto é, o conhecimento), na relação educativa com os residentes multiprofissionais, que é oriunda de processos sistemáticos.

\section{A percepção dos demais atores sociais da RMS sobre a capacitação do enfermeiro para o desempenho de suas funções na preceptoria}

No que concerne à capacitação, também os residentes avaliaram que o enfermeiro-preceptor, agente social fundamental para sua formação no campo prático, precisa passar por atualizações. Indicaram a 
necessidade de revisão não das questões didáticas, metodológicas, pedagógicas ou curriculares, mas, dos saberes inerentes à profissão e ou à esfera de atuação, para avanços na construção do conhecimento nos momentos com eles. Apresentaram temas que o enfermeiro pode desenvolver com qualquer residente, independente da categoria, como por exemplo, o conceito de clínica ampliada, que abrange o reconhecimento da singularidade do sujeito, a integralidade da assistência, a humanização, o uso da intersetorialidade e tantos outros preconizados pelo SUS para a saúde pública.

"Muitas enfermeiras são ainda de uma formação antiga, antes desse conceito ampliado de saúde e tudo mais. Mas, nós precisamos nos atualizar, então a preceptoria é importante para formação [...], sem preceptor o residente não deveria nem estar no campo. Mas, nós precisamos ter preceptores que realmente façam um papel interessante numa discussão de caso, numa reflexão de textos no campo teórico e no campo prático, para que possa encaminhar o residente, porque o que é bom para o residente é bom para o enfermeiro, que também tem essa função" (R18Odontologia).

"E eu acho também que os enfermeiros daqui, que são preceptores, eles precisavam passar por uma reciclagem, porque muitas coisas já estão ultrapassadas e eles acabam passando uma coisa que nem faz mais" (R6-Enfermagem).

Um estudo apontou que as categorias profissionais que compõe os programas de residência multiprofissionais em saúde ganham destaque, onde grande parte das profissões já se apresentam com porcentagens importantes, evidenciando-se a enfermagem com mais de $90 \%$ de participação nos processos (SILVA MB, et al., 2021). Contudo, mesmo que tenha um número expressivo de enfermeiros nos programas existem outras categorias e há necessidade de também se capacitar para o ensino interprofissional que deve ser reflexo de um trabalho interprofissional da unidade de saúde do SUS, vivido intensamente pelo preceptor (ARAÚJO TAM, et al., 2017).

"Nas clínicas da família, no geral, você não tem uma formação de preceptoria tão desejada assim. [...] E o que eu observo é que nós tivemos uma enfermeira que foi nossa preceptora mas que não sabia o que é "preceptorar". [...] Eu tive a figura de uma enfermeira que queria ser preceptora, mas que não "preceptorou" por não saber como" (R18 - Odontologia).

O domínio dos conhecimentos e as experiências da profissão para acompanhar, orientar, avaliar e supervisionar o treinamento permanente em serviço dos residentes deve estar atrelado aos saberes básicos do campo educacional, a fim de que o enfermeiro-preceptor também seja empoderado para discutir sobre os nós da residência, fazer críticas pertinentes, dar sugestões, participar no desenvolvimento de trabalhos científicos e ampliar sua participação como protagonista no programa.

Leite MSBS, et al. (2020) indicaram que enfermeiros assistenciais, que atuam como preceptores, precisam de capacitação pedagógica para possibilitar sempre a troca de experiências e conhecimentos nos espaços de produção do cuidado em saúde.

Compreender quais são as atribuições previstas nas publicações específicas do Ministério da Educação e Ministério da Saúde e o que é esperado pelos programas, e estes orientados pelos Projetos Políticos Pedagógicos, manuais e regimentos, deveria orientar a atuação do preceptor, mas isto depende minimamente do interesse em procurar a informação e auxílio nos casos de não entendimento amplo do que está registrado nas orientações do curso (BRASIL, 2010; BRASIL, 2006; BRASIL, 2010).

A Resolução no 2 de 13 de abril de 2012, destaca que compete a coordenação "constituir e promover a qualificação do corpo de preceptores", mas, não esclarece com detalhes como isso deve ser posto em prática (BRASIL, 2012). Como lemos, no depoimento a seguir, a coordenadora auxiliou um preceptor, dialogando com ele, fornecendo materiais para estudo e indicando cursos:

"Passei para esse preceptor alguns instrumentos, alguns textos para ele avaliar, para melhorar na formação. Enfim, tivemos uma conversa bem sincera acerca da preceptoria e indiquei meios que ela pudesse estar se inteirando mais da questão da residência, cursos de formação e de preceptoria" (C3). 
A equipe de coordenação poderia disponibilizar uma cópia de cada documento organizados em uma pasta nas unidades que recebem residentes e comunicar ao profissional, como uma forma de acesso rápido aos materiais, colocando-se a disposição para esclarecimento de dúvidas. Isso já reduziria a exposição dele a julgamentos, que de alguma maneira sempre existirão, seja da parte dos educandos ou de outros atores sociais envolvidos com as atividades da RMS.

Indica-se também a realização de reuniões e encontros com os tutores, docentes, coordenadores e os próprios residentes, mas as demandas da rotina de trabalho nem sempre permitem a organização destes e o preceptor não consegue sair do espaço do serviço para efetivamente se envolver; sendo, portanto, necessário planejamento prévio e combinação de agendas para facilitar a participação de todos envolvidos.

O depoimento da tutora converge com o das enfermeiras-preceptoras, indicando que a capacitação deveria ser realizada desde o início da atuação nos programas, reconhecendo que assumem a tarefa de ensinar sem o preparo necessário. Destacam-se os temas: como se relacionar com estudantes e como utilizar os dispositivos educacionais, como ensinar o que se pratica até mesmo de forma mais exemplar e como conduzir o residente à crítica profissional nas transformações positivas tão esperadas no mundo do trabalho.

"[...] tem que ser uma pessoa que tem que passar primeiro por um treinamento, uma capacitação talvez, um treinamento para saber como lidar. Porque nem todo mundo que está na prática tem um conhecimento [...] aquela coisa assim mais refinada de como vou lidar com aluno, como é que eu vou passar informação, como é que eu vou transmitir esse conhecimento [...] muitas pessoas lá no campo sabem muito, mas não sabem como fazer isto [...]" (T3).

Ao levantar os problemas a partir da prática dos enfermeiros na preceptoria, há que se ter coragem para lutar, pondo-se à caminho da instrumentalização para melhor desempenho da atividade educativa na RMS. Para tal se faz importante a educação permanente e deve-se contar com todo apoio dos demais membros partícipes deste programa, em especial da coordenação, dos docentes e dos tutores, que atuam como educadores igualmente (BRASIL, 2009).

Finalmente, fazendo uma apropriação dos escritos de Saviani D (2011) sobre a formação dos professores, é interessante considerar que "uma boa formação se constitui em premissa necessária para 0 desenvolvimento de um trabalho docente qualitativamente satisfatório.". Ou seja, um enfermeiro-preceptor bem preparado tem condições de realizar adequadamente a tarefa educativa dentro do seu espaço de atuação assistencial, que se traduza em um trabalho de qualidade tanto para os usuários quanto para os estudantes multiprofissionais e demais atores da residência em saúde.

\section{CONCLUSÃO}

Por meio do depoimento de diferentes atores sociais da RMS, o estudo evidenciou a importância de valorizar a atuação do enfermeiro na preceptoria dos distintos programas, a quem deve-se oportunizar tempo formal de capacitação que dê subsídios para um trabalho educativo junto aos residentes nos serviços atual, relevante, crítico e transformador das práticas de saúde pública, onde todos aprendem permanentemente.

\section{REFERÊNCIAS}

1. ARAÚJO TAM, et al. Multiprofessionality and interprofessionality in a hospital residence: preceptors and residents' view. Interface (Botucatu), 2017; 21(62): 601-613.

2. BRASIL. Conselho Nacional de Saúde. Resolução № 287 de 08 de outubro de 1998. Relaciona as categorias profissionais de saúde de nível superior para fins de atuação no CNS. Diário Oficial da União, Brasília/DF, 1998. Disponível em: http://www.crefrs.org.br/legislacao/pdf/resol_cns_287_1998.pdf. Acessado em: 25 de março de 2021.

3. BRASIL. Secretaria geral a Presidência da República. Consēho Naciōnal de Juventude. Lei no 11.129 , de 30 de junho de $2005 . \quad$ Brasília/DF, 2005. Disponível em: http://portal.mec.gov.br/index.php?option=com_content\&view=article\&id=12500\%3Alegislacaoespecifica\&catid=247\%3Aresidencia-medica\&Itemid=813. Acessado em: 24 de junho de 2014.

4. BRASIL. Ministério da Saúde. Secretaria de Gestão do Trabalho e da Educação na Saúde. Departamento de Gestão da Educação na Saúde. Residência multiprofissional em saúde: experiências, avanços e desafios. Brasília, 2006. Disponível em: http://bvsms.saude.gov.br/bvs/publicacoes/residencia_multiprofissional.pdf. Acessado em: $18 \mathrm{de}$ março de 2021. 
5. BRASIL. Ministério da Saúde. Secretaria de Gestão do Trabalho e da Educação na Saúde. Departamento de Gestão da Educação em Saúde. Política Nacional de Educação Permanente em Saúde. 2009. Disponível em: http://bvsms.saude.gov.br/bvs/publicacoes/pacto_saude_volume9.pdf. Acessado em: 18 de março de 2021.

6. BRASIL. Ministério da Educação. Portaria interministerial MEC/MS no1.320, de 11 de novembro de 2010. Dispõe sobre a estrutura, organização e funcionamento da Comissão Nacional de Residência Multiprofissional em Saúde - CNRMS. Brasília: 2010. Disponível em: http://portal.mec.gov.br/index.php?option=com_docman\&view=download\&alias=15466port-1320-11 nov-2010\&ltemid=30192. Acessado em: 18 de março de 2021.

7. BRASIL. Ministério da Saúde. Grupo Hospitalar Conceição Residências em saúde: fazeres \& saberes na formação em saúde; organização de Ananyr Porto Fajardo, Cristianne Maria Famer Rocha, Vera Lúcia Pasini. Porto Alegre: Hospital Nossa Senhora da Conceição, 2010. 260 p.

8. BRASIL. Resolução no 2, de 13 de abril de 2012. Dispõe sobre Diretrizes Gerais para os Programas de Residência Multiprofissional e em Profissional de Saúde. Brasília, 2012. Disponível em: http://www.in.gov.br/visualiza/index.jsp?data=16/04/2012\&jornal=1\&pagina=24\&totalArquivos=168. Acessado em: 10 de novembro de 2012.

9. BRASIL. Ministério da Saúde. Conselho Nacional de Saúde. Resolução nํ466, de 12 de dezembro de 2012. Dispõe sobre pesquisa envolvendo seres humanos. Brasília (DF): MS, 2012.

10. FRANCO TB, MERHY EE. Cartografias do Trabalho e Cuidado em Saúde. Tempus. Actas de Saúde Coletiva, 2012; 6 (2): 151-163.

11. LEITE MSBS, et al. Percepção dos enfermeiros sobre desenvolvimento de competências durante a residência. Revista Eletrônica Acervo Saúde, 2020; 12(10): e4121.

12. MELLO AL, et al. Formação de Residentes Multiprofissionais em Saúde: Limites e Contribuições para a Integração Ensino-Serviço. Revista de Enfermagem do Centro Oeste Mineiro, 2018; 8: e2567.

13. MINAYO MCS (org.). Pesquisa Social: Teoria, Método e Criatividade. 27. ed. Rio de Janeiro: Vozes, 2007; 108p.

14. RIBEIRO PKC, et al. Os profissionais de saúde e a prática de preceptoria na atenção básica: assistência, formação e transformações possíveis. J Manag Prim Health Care, 2020; 12: e21.

15. SAVIANI D. Pedagogia Histórico-Crítica: primeiras aproximações. 9. ed. São Paulo: Autores associados, 2005, 160p.

16. SAVIANI D. As Concepções Pedagógicas na História da Educação Brasileira. Campinas: 2005. Disponível em: https://www.histedbr.fe.unicamp.br/pf-histedbr/dermeval_saviani_artigo.pdf. Acessado em: 18 de março de 2021.

17. SAVIANI D. História das ideias pedagógicas no Brasil. 3.ed. rev. Campinas, São Paulo: Autores Associados, 2010. $595 p$.

18. SAVIANI D. Interlocuções pedagógicas - conversa com Paulo Freire e Adriano Nogueira e 30 entrevistas sobre educação. Campinas, São Paulo: Autores Associados, 2010. 296p.

19. SAVIANI D. Ciência e educação na sociedade contemporânea: desafios a partir da pedagogia histórico-crítica. Revista Faz Ciência, 2010; 12(16): 13-36.

20. SAVIANI D. Formação de professores no Brasil: dilemas e perspectivas. Poíesis Pedagógica, $2011 ; 9$ (1): $07-19$.

21. SAVIANI D. A pedagogia histórico-crítica. Revista RBBA, 2014; 3 (2): 11-36.

22. SILVA MB, et al. Caracterização das residências multiprofissionais em saúde do Brasil. Revista Eletrônica Acervo Saúde, 2021;13(2): e5491.

23. SILVA VC, et al. Prática social e pedagógica do enfermeiro-preceptor: um estudo de caso. Online Brazilian Journal of Nursing, 2014; 13 (1): 102-112.

24. SILVA VC. Os saberes que emergem da prática social do enfermeiro- preceptor na residência multiprofissional em saúde. Tese (Doutorado em Enfermagem) - Escola de Enfermagem Anna Nery. Universidade Federal do Rio de Janeiro, Rio de Janeiro, 2017; 197p.

25. SOUZA SV, FERREIRA BJ. Preceptoria: perspectivas e desafios na Residência Multiprofissional em Saúde. ABCS Health Sci, 2019; 44(1):15-21. 